Article

\title{
Hybrid Nanosystems Based on Metal-Containing Mesogenic CyanoAlkyl and Alkoxybiphenyls
}

\author{
Tatyana I. Shabatina $1,2, * \mathbb{B}$ and Yurii N. Morosov 1,2 \\ 1 Department of Chemistry, M.V. Lomonosov Moscow State University, Leninskie Gori build 1/3, \\ 119991 Moscow, Russia; yunmor@mail.ru \\ 2 Bauman Moscow State Technical University, 2nd Bauman Str. 5, 105905 Moscow, Russia \\ * Correspondence: tatyanashabatina@yandex.ru or tsh@kinet.chem.msu.ru; Tel./Fax: +7-495-939-5442
}

Received: 18 December 2019; Accepted: 15 January 2020; Published: 30 January 2020

check for updates

\begin{abstract}
The paper reviews the results of the authors on the production of hybrid nanosystems based on liquid crystalline (LC) long-chain cyano(alkyl and alkoxy)biphenyls (5CB, 5OCB, and 8CCB) including nanosized metal species. The samples were obtained through the direct incorporation of metal (silver and copper) atoms and small clusters into mesogenic CB matrices via a low temperature co-condensation technique, and the formation of biligand metal complexes were revealed by FTIR and ESR-spectroscopy. The heating of the systems led to the controlled growth of metal clusters and nanosized metal particles of the definite size beginning from 1 up to 200 nanometers, and their highly-ordered assemblies stabilized in the solid and liquid crystalline phases. It is shown that supramolecular ordering in different LC phases of cyanobiphenyl matrices determines the size and shape of nanosized metal species that are formed in the systems under investigation, as well as the morphology of their aggregates. TEM and atomic force microscopy (AFM) data revealed the existence of orientationally-ordered nanostructures in the nematic phases of 5CB and 5OCB. The growth of quasi-fractal 2D-aggregates was shown for layer-structured smectic mesophase of $8 \mathrm{CB}$. The UV-Visible spectra of hybrid metal-mesogenic nanosystems $\mathrm{Ag}-5 \mathrm{CB}$ and $\mathrm{Cu}-5 \mathrm{CB}$ that were incorporated into polymeric films revealed intensive plasmonic bands at $400-450 \mathrm{~nm}$, similar to silver nanoparticles, and 540-650 nm, similar to copper nanoparticles. The increasing of the metal contents in the samples caused the growth of highly anisometric shaped metal rods, with the ratio of the length to the diameter being more than 10 and plasmonic bands at region of $\lambda \geq 650 \mathrm{~nm}$.
\end{abstract}

Keywords: Cyanoalkyl(alkoxy)biphenyls; metal-mesogenic nanosystems; antiferroelectric ordering; hybrid plasmonic nanostructures; silver nanoparticles; copper nanoparticles; controlled self-organization; optical properties

\section{Introduction}

Hybrid nanosystems, including nanosized metal particles that are incorporated in structural organic matrices (liquid crystals, polymers, etc.) have gained the rising interest of researchers in different areas due to their possible applications in molecular electronics, nanophotonics, catalysis and sensor systems [1-9]. The use of liquid crystalline matrices as soft, highly-ordered templates expands the possibilities for the synthesis of anisometric metal nanoparticles and the formation of highly-ordered nanostructures. Such systems combine the unique quantum size properties of nanosized objects with the possibility of their spatial.

The organization of nanosized metal particles in highly-ordered assemblies and the creation of hybrid nanostructures with definite structural and morphological characteristics is nowadays one of the most promising areas of nanoscience and nanotechnology [3-5]. The unique quantum-size properties and the appearance of new collective modes have opened new possibilities of their application in 
such fields as microelectronics, optics, catalysis, information recording systems, and data storage systems [7-10]. The physical and chemical properties of such nanohybrid systems depend on the size and shape of the structural elements (nanoclusters and nanoparticles), as well as on the manner of their organization into the joint highly-ordered structures [11,12]. The development of new synthesis methods of nanohybrid aggregates that possess a special order in the regular placement of the particles of the same size is an important task in modern nanochemistry.

The inclusion of metal ions, atoms and small clusters in liquid crystalline media changes the whole system of van der Waals and dipole-dipole intermolecular interactions, but it also leads to the production of donor-acceptor complexes of different nuclearity of metal species. This is followed by the appearance of color and magnetic properties of the whole hybrid system. The most prospective among such objects are hybrid nanosystems, including nanosized metals that possess unique quantum-sized chemical and physical properties and that are extremely different from metal atoms, metal clusters, and bulk materials with the possibility of their 2D and 3D space ordering due to their interaction with the molecules of self-assembled mesogenic matrix compounds. The unique characteristics of such hybrid systems are determined not only by the morphology of metal particles and their aggregates that are incorporated in a liquid crystalline matrix but also by their joint spatial self-organization by a liquid-crystalline matrix [10-20].

The application of the cryogenic vacuum condensation [21,22] of metal atoms and organic molecular beams on the cooled supporting surfaces leads to the formation of metal clusters and their metastable complexes. By this method, we could use a bottom up approach that begins from metal atoms and individual and dimeric cyanobiphenyl molecules in order to produce highly-ordered hybrid nanosystems by the self-assembling process that is controlled by the structural and dynamic properties of liquid-crystalline matrices.

In this work, the formation of new hybrid metal-mesogenic nanosystems is made on the example of two d-bock metals of the 11 group-silver and copper with mesogenic derivatives of long-chain alkyl and alkoxy cyanobiphenyls. Silver and copper nanoparticles possess intensive plasmonic bands in the visible region of the spectrum and can be used for the visualization and marking of different biological objects. The mesogenic compounds of the alkyl(alkoxy)cyanobiphenyl row are nowadays widely used as components of active layers for liquid crystalline displays and indicators. These compounds form ordered liquid crystalline phases of two types: nematic, characterized by the existence of molecular orientations long range order, and smectic, with layered molecular ordering. The existence of molecular associates and the formation of dimeric structures in solid and liquid crystalline phases is known for these compounds at low temperatures. They form several metastable and stable solid phases with local structures similar to liquid crystalline mesophases by with different molecular organizations [23,24]. The terminal cyano-group and aromatic core of the molecules can be considered suitable for the formation of complexes of $\pi$ and $\sigma$ types with the atoms and clusters of the metals that are under consideration [25].

The aim of this work was focused on the development of prospective methods of new hybrid nanosystem creation and the formation of hybrid metal-mesogenic nanostructures by using the self-organized liquid crystalline matrices. The mechanisms and roles of the different donor-acceptor interactions of the surface centers of metal species and the functional groups of organic molecules, as well as their effects on the intermolecular dipole-dipole interactions and self-organization processes, are considered. The new physico-chemical properties that appear for the obtained hybrid metal-mesogenic nanosystems are demonstrated with a combination of silver and copper metal nanoparticles that are formed in mesogenic matrices of long chain $(n \geq 5)$ cyanoalkylbiphenyls: 4-cyano-4'-pentylbiphenyl (5CB) and 4-cyano-4'-octylbiphenyl $(8 \mathrm{CB})$ and cyanoalkoxybiphenyl: 4-cyano-4'-pentyloxybiphenyl (5OCB). 


\section{Materials and Methods}

\subsection{Materials}

The 5CB, 8CB, and 5OCB liquid crystals that were produced by Merck (Merck KGaA, Darmstadt, Germany) (99\%) were used without further purification.

\subsection{Samples Preparation}

Metal-containing film samples containing liquid crystalline compounds and nanometals were obtained through the co-condensation of the components on a surface that was cooled with liquid nitrogen in the regime of molecular beams. The transfer of substances to the gas phase was carried out by resistive heating. To register the transmission UV-Vis spectra, we used vacuum spectral cryostats that were equipped with cooled spectral windows made from quartz, $\mathrm{KBr}$, and $\mathrm{CaF}_{2}$. To obtain samples for ESR spectroscopy, the co-condensation of the components was carried out on a quartz tube, which was placed into the resonator of the ESR spectrometer with a movable vacuum connection. The design of the cryostats made it possible to conduct the spectroscopic examination of the film samples in situ under vacuum conditions, with a thermostatic control in the temperature range of 80-320 K. The metal atoms' flow was set by raising the temperature of the resistive heater. The calibration of the dependence of the metal flow on temperature was carried out by using a special procedure along with a quartz micro weighting technique, i.e., the dependence of the natural frequency of the quartz microcrystal resonator on the mass of the metal deposited on its surface. The absolute value of metal was determined by photometric analysis and ICP mass spectrometry. The metal-ligand ratio in the samples was changed from 1:1 to 1: $10 \mathrm{~mol}$.

\subsection{Characterization Techniques}

Spectral investigations of metal atoms complexation and of the supramolecular ordering of the systems including silver and copper were obtained by the high vacuum cryocondensation of metal and mesogenic compound vapors on the cooled spectral surfaces of different cryostats (polished copper, quartz, $\mathrm{KBr}$, or $\mathrm{CaF}_{2}$ depending on the type of spectral procedure) under molecular beam conditions.

\subsubsection{UV-Vis}

A Specord M-40 UV-visible absorption double beam spectrophotometer (Carl Zeiss Jena, Germany) in transmission mode was used to scrutinize the spectra.

\subsubsection{ESR-Spectroscopy}

The ESR spectra were registered with the X-band ESR spectrometer (model P-1307) at a $100 \mathrm{kHz}$ modulation field of a $1 \mathrm{Gs}$ amplitude All equipment functioned under a computer program suit in the OS-2 Warp 4.0 operating system.

\subsubsection{Transmission Electron Microscopy}

Transmission electron microscopy (TEM) images were recorded with a LEO 912 AB Omega ZEISS electron microscope (ZEISS, Oberkochen, Germany) with an electron accelerating voltage of $100-200 \mathrm{kV}$.

\subsubsection{Atomic Force Microscopy}

Atomic force microscopy (AFM) images were recorded with Integra Spectra II (NT MDT, Moscow, Russia). 


\subsubsection{X-ray Powder Diffraction}

X-ray powder diffraction patterns were recorded by Empyrian (PAN analytical, Almelo, Netherlands) ( $\mathrm{Cu} \mathrm{K} \alpha$ radiation, Ni $\beta$-filter, Bragg-Brentano mode).

\section{Results and Discussion}

\subsection{Production of the Metal Clusters}

Long-chain cyano(alkyl-) or (alkoxy)biphenyls formed in the liquid crystalline and crystal solid states dimeric supramolecular structures due to strong dipole-dipole interactions and the anti-ferroelectric arrangement of the molecules by the head-to-tail principle. The characteristic size of such structures was $2.57 \mathrm{~nm}$ for the molecular dimer $5 \mathrm{CB}$ molecules. The introduction of metal atoms (silver or copper) in a cyanobiphenyl matrix $(\mathrm{CB})$ were been made by the cryogenic co-condensation of metal atomic vapor, and the molecular beam of the mesogenic compounds $(5 \mathrm{CB}, 8 \mathrm{CB}$, and $5 \mathrm{OCB}$ ) led to the formation of bi-ligand complexes structures in which a between-metal atom was embedded in the molecular dimer structure between the $\mathrm{CN}$-group of one $\mathrm{CB}$ molecule and the phenyl ring of the other CB molecule arranged by the head-to-tail principle (Figure 1). The distribution of the electron density in the complex structure in the case of two border electronic configurations that were obtained by the DFT/B3LYP modelling of the system is shown in Figure S2.

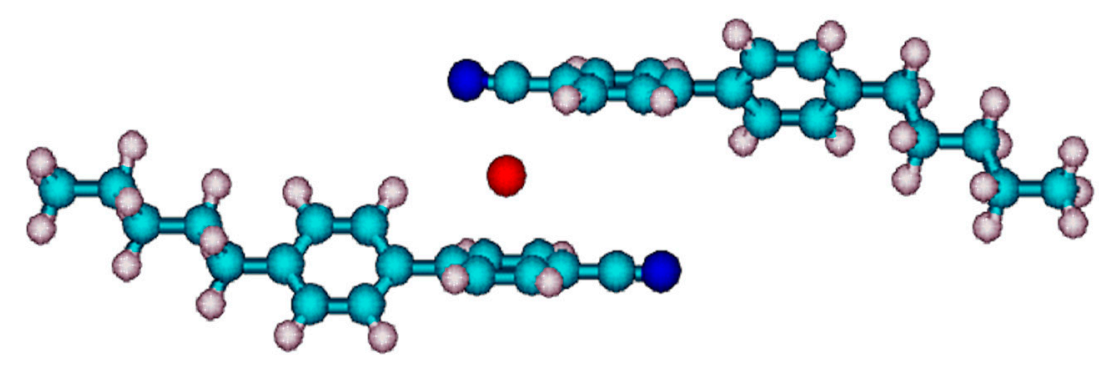

Figure 1. The scheme of a 4-cyano-4'-pentylbiphenyl (5CB) bi-ligand molecular complex $\mathrm{Ag}(5 \mathrm{CB})_{2}$.

The appearance of new bands in the region of the triple $\mathrm{CN}$ bond stretching vibrations at 2030 and $2080(2130) \mathrm{cm}^{-1}$ was recorded in the IR-Fourier spectra of the Ag-cyanobiphenyl $(5 \mathrm{CB}, 8 \mathrm{CB}$, and $5 \mathrm{OCB}$ ) samples due to the formation of biligand $\pi$-complexes of the metals in the zero oxidation state with cyanobiphenyls. The existence of two different bands for $\mathrm{CN}$ oscillations indicated the nonequivalence of the two $\mathrm{CN}$ groups in the structure of the biligand complex. In the IR-Fourier spectra of the samples, another new band was also recorded at $650-660 \mathrm{~cm}^{-1}$, this one related to the oscillations of the metal-ligand bonds in the $\pi$-complex. The Ag-CB complexes were stable at a temperature of $77 \mathrm{~K}$, but they were completely decomposed when the temperature rose to 150-200 K.

For the ESR spectroscopy studies at low temperatures and high vacuum conditions, we used a cryostat of a special design, the scheme of which is shown in Figure S1. The cryostat allowed for the simultaneous co-condensation of three substances: metal $(\mathrm{Ag}, \mathrm{Cu})$, cyanobiphenyl $(5 \mathrm{CB}, 8 \mathrm{CB}$, and $5 \mathrm{OCB}$ ) and the third reactive component $\left(\mathrm{CCl}_{4}\right)$ in the molecular (atomic) beam mode.

The ESR spectra of double co-condensates $\mathrm{Ag}$ with $5 \mathrm{CB}$ and $8 \mathrm{CB}$ that were obtained and registered at a temperature of $77 \mathrm{~K}$ contained three groups of signals (A, B and C) (Figure 2). Doublet signals (A, B) in the region of higher and lower magnetic fields belonged to the biligand $\pi$-complexes $\operatorname{Ag}(C B)_{2}[22]$. The formation of the doublet was caused by the difference in the magnetic resonance parameters of the $\mathrm{Ag}^{107}$ and $\mathrm{Ag}^{109}$ isotopes that were contained in the natural silver in an approximately equivalent ratio. The values of the magnetic resonance parameters of these signals that were obtained by the computer processing of experimental spectra are typical for the $\pi$-complexes of silver atoms. In these complexes, the main contribution to the formation of the molecular orbital on which the unpaired electron is located was introduced by the 5s-orbital of the silver atom. A comparison of the values of the isotropic hyperfine interaction constants of silver atoms that are part of biligand complexes with 
values that are typical for undisturbed silver atoms [26] allowed us to estimate the spin density of the s-electron of the silver of the $\mathrm{Ag} / 5 \mathrm{CB}$ system as $\sigma_{\mathrm{M}}=0.89$. This value is typical for the $\pi$-complexes of silver atoms and shows the degree of partial electron density donation from the silver atom to the system of the $\pi^{*}$-orbitals of ligand molecules.

There was also a central asymmetric singlet ESR signal (C) with a g-factor close to the value of 2.0032 - the g-factor of the free electron - present spectra of these systems. This signal can be attributed to the spin resonance of the conduction electrons of nanoscale silver clusters [22]. By using the method of ESR spectroscopy, it is possible to trace the kinetics of the degradation of biligand complexes and the formation of nanoscale silver clusters when the temperature increases.

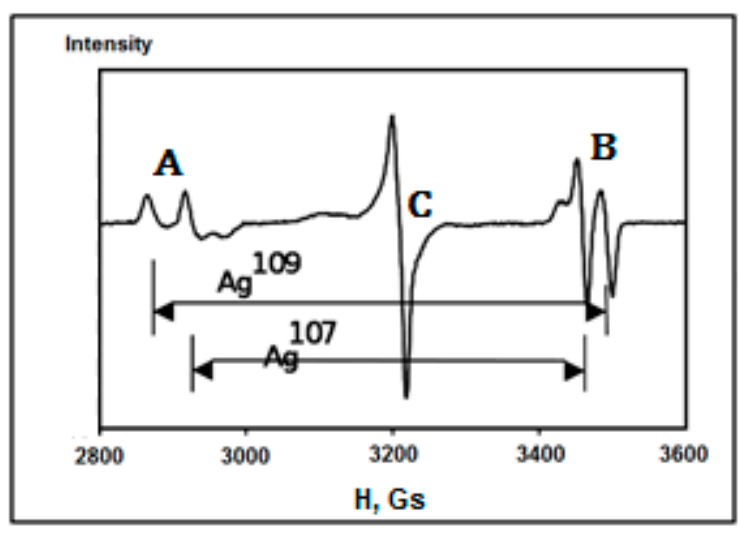

(a)

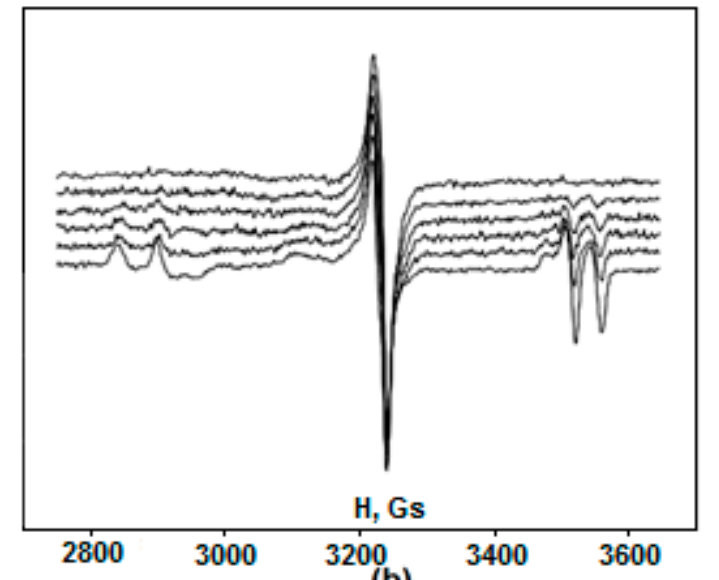

(b)

Figure 2. ESR spectra of silver-cyano-4'-pentylbiphenyl co-condensate film (a) at $80 \mathrm{~K}$ and (b) at different temperatures of, from bottom to top, 80, 108, 120, 140, 150, 182, and $200 \mathrm{~K}$.

When heating the samples of the double $\mathrm{Ag} / \mathrm{CB}$ co-condensates from 77 to $150{ }^{\circ} \mathrm{C}$, the relative integral intensity of the doublet signals A and B gradually decreased, and the intensity of the central singlet signal $C$ simultaneously increased. This indicates the thermal destruction of the biligand $\pi$ complexes $\mathrm{Ag}(\mathrm{CB})_{2}$. The liberated silver atoms acquired the ability to diffuse relatively freely within a solid-phase matrix, which was an unordered glassy liquid-crystalline phase. Due to diffusion, silver atoms either combined to form new nanoclusters or were incorporated into existing ones. As a result, the integral intensity of the spin resonance signal of the conduction electrons (C) increased. In the temperature range of $150-200 \mathrm{~K}$, the intensity of this signal remained almost constant (taking into account the dependence of the signal intensity on the temperature). Heating samples above $200 \mathrm{~K}$ led to the crystallization of a glassy liquid crystal matrix and a sharp increase in molecular mobility, thus resulting in the rapid coalescence of nanoclusters to form aggregates that did not give a signal in the ESR spectra.

The addition of the third active component to the system $-\mathrm{CCl}_{4}$, in our case-in an equimolar ratio with respect to silver led to an increase in the concentration of the $\pi$-complexes of silver atoms with cyanobiphenyl molecules (components A and B in Figure 2). At the same time, the central signal in the $\mathrm{Ag} / 5 \mathrm{CB} / \mathrm{CCl}_{4}$ triple system acquired a more symmetrical shape compared to that in the $\mathrm{Ag} / 5 \mathrm{CB}$ double system (Figures 3 and 4). Increasing the temperature in the double system to 170-200 K led to an increase of the intensity of the central component and to the appearance of a highly symmetric signal with a g-factor close to the value of the g-factor of the free electron (Figure 4f,g,h) in the ESR spectra. 


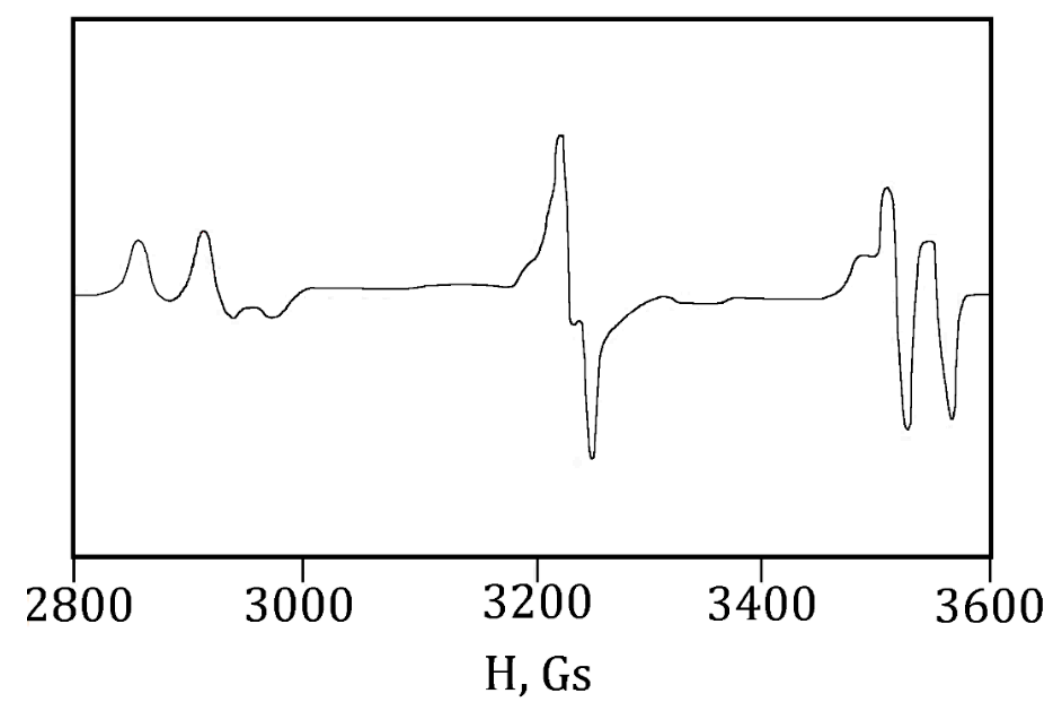

Figure 3. ESR spectrum of sample of the $\mathrm{Ag} \div \mathrm{CCl}_{4} \div 4$-cyano- $4^{\prime}$-pentylbiphenyl (1:1:100 mol. \%) triple system that was obtained during vacuum co-condensation at a temperature $77 \mathrm{~K}$.

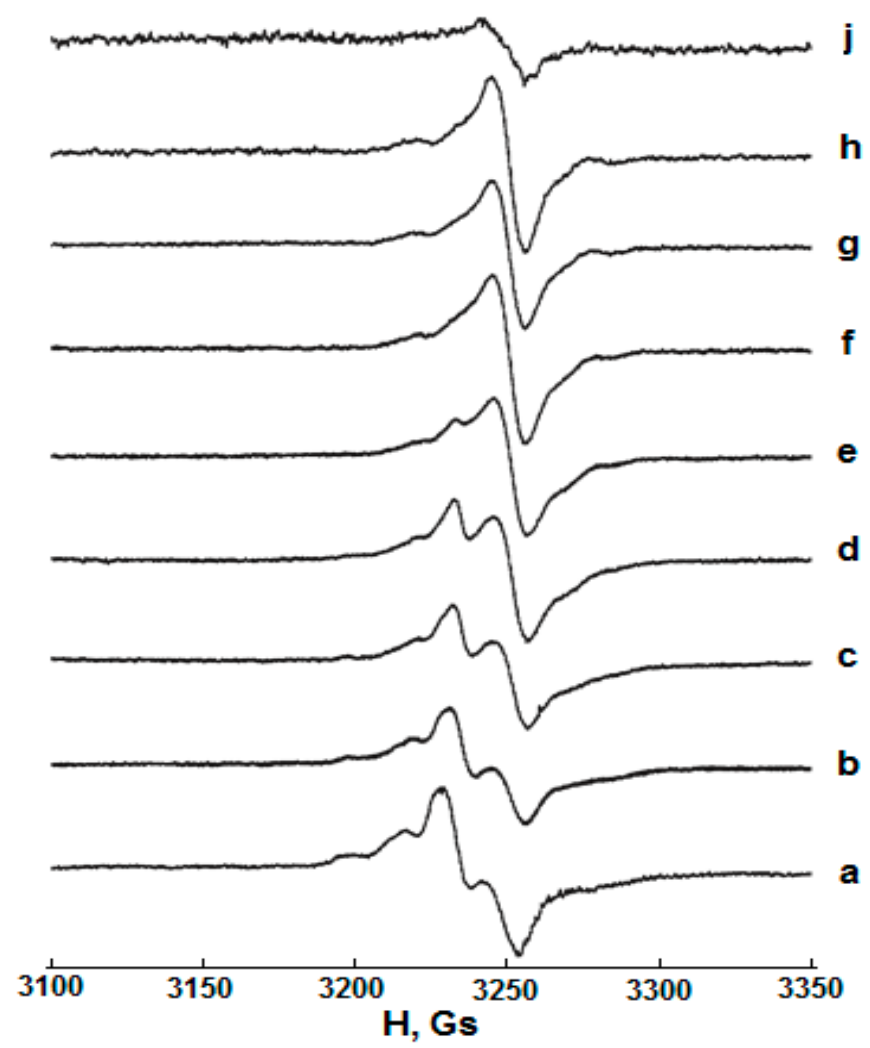

Figure 4. Central part of the ESR spectra of sample of the $\mathrm{Ag} \div \mathrm{CCl}_{4} \div 4$-cyano-4'-pentylbiphenyl $(1: 1: 100 \mathrm{~mol} . \%)$ triple system that was obtained during vacuum co-condensation and was registered after heating to the following temperatures: $a-77 \mathrm{~K} ; \mathrm{b}-100 \mathrm{~K}$; $\mathrm{c}-120 \mathrm{~K}$; d-140 K; e-160 K; f-180 K; g-190 K; h-200 K; and j-210 K.

The observed effects can be explained by the fact that silver atoms and small silver clusters have different reactivities in relation to the molecule $\mathrm{CCl}_{4}$. The highly symmetric icosahedral $\mathrm{Ag}_{13}$ clusters did not interact with $\mathrm{CCl}_{4}$, and, at the same time, a set of other asymmetric small silver clusters, which gave an ESR signal in the central region of the spectrum, reacted with this molecule. In addition, small clusters of silver with an even number of atoms $\left(\mathrm{Ag}_{2}\right.$ and $\left.\mathrm{Ag}_{4}\right)$ do not have unpaired electrons and, therefore, did not give a signal in the ESR spectra. When co-condensing under 
conditions of high molecular mobility, small silver clusters could react with the $\mathrm{CCl}_{4}$ molecule to release silver atoms. This caused an increase in the concentration of the $\pi$-complexes of the silver atoms with cyanobiphenyl molecules in the triple system. Increasing the temperature led to the thermal degradation of the biligand $\pi$-complexes of the silver atoms with the dipolar dimer of the CB-molecules and the rising of the diffusion mobility of the silver atoms. The silver atoms themselves interacted with $\mathrm{CCl}_{4}$ molecules, forming the reaction product $\mathrm{AgCl}$ and nonstable silver clusters, which could decompose and form silver atoms that aggregated at the next stage in order to form non-reactive, highly symmetric icosahedral $\mathrm{Ag}_{13}$ and $\mathrm{Ag}_{55}$ clusters.

The ESR spectra of the samples of copper-cyanobiphenyl (Cu-5CB) double co-condensates, obtained at a temperature of $77 \mathrm{~K}$, contained two signals. The first was a four-component signal (signal A in Figure 5) that was caused by the formation of the copper-cyanobiphenyl $\pi$-complex (the nuclear spin of copper is 3/2). This signal was characterized by a pronounced anisotropy of magnetic resonance parameters. The value of the perpendicular component of the hyperfine interaction constant (HFI) $\mathrm{B} \approx 51 \mathrm{MHz}$. Additional splitting due to the presence of two isotopes in natural copper $\left(\mathrm{Cu}^{63}\right.$ and $\left.\mathrm{Cu}^{65}\right)$ was not allowed due to the proximity of their nuclear g-factors. A comparison of this value with the value $\mathrm{B}_{0}=386 \mathrm{MHz}$, typical for an electron that is located on the 4p-orbital of an undisturbed copper atom [27], allowed us to determine the value of the spin density of an unpaired electron on the $4 \mathrm{p}_{z}$ orbital of a copper atom in the copper-cyanobiphenyl $\pi$-complex; that value was $\sigma_{M}=0.13$. The process of the jumping an unpaired electron of a copper atom from a $4 \mathrm{~s}$ to a $4 \mathrm{p}$ orbital is known for biligand $\pi$-complex copper atoms in the oxidation state of zero with organic ligands [27].

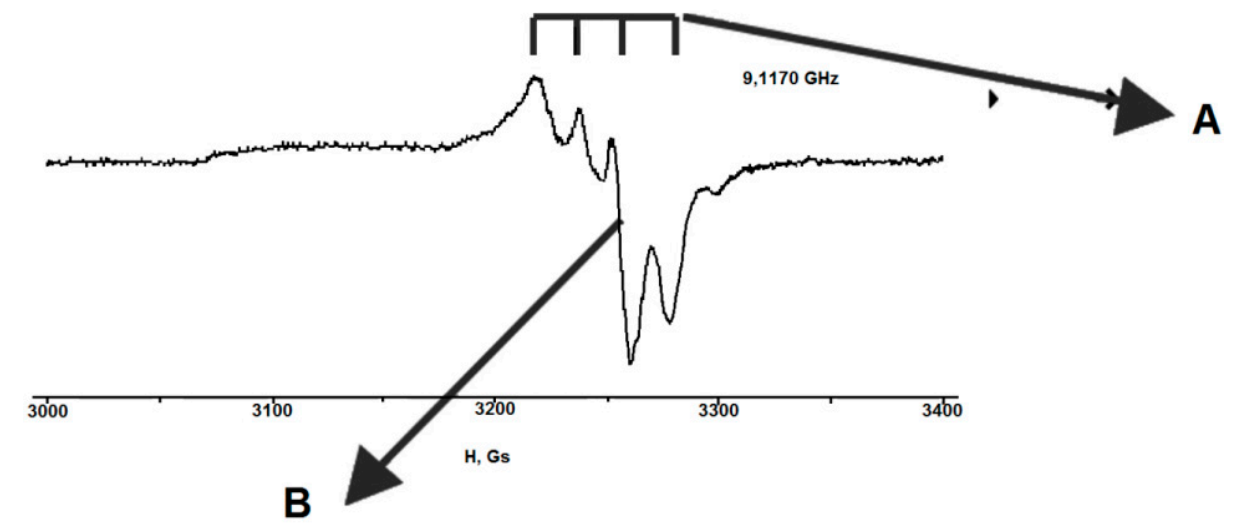

Figure 5. ESR spectra of a Cu-4-cyano-4'-pentylbiphenyl co-condensate film at $77 \mathrm{~K}$ (Y-axis is the intensity of ESR-signal in related unites).

Another component of the ESR spectrum of the copper-cyanobiphenyl double co-condensate $(\mathrm{Cu} / 5 \mathrm{CB})$ (signal B in Figure 5) represented a singlet with a g-factor close to the value of 2.0032-the $\mathrm{g}$-factor of the free electron. This was caused by the spin resonance of the conduction electrons of the nanoscale copper clusters. The increase in temperature led to the thermal decomposition of the biligand complexes of the copper atoms and the additional formation of nanoscale copper clusters.

The study of film samples of hybrid nanosystems "silver-4-cyano-4-pentylbiphenyl" (Ag-5CB), "silver-4-cyano-4-pentyloxybiphenyl"(Ag-5OCB) and "copper-4-cyano-4-pentylbiphenyl" (Cu-5CB) by transmission electron microscopy showed the formation and stabilization of spherical metal nanoparticles in a glassy-like frozen liquid crystal matrix with a size of 1-2 $\mathrm{nm}$ (Figure 6). These samples were obtained by the co-condensation of the components on a surface that was cooled with liquid nitrogen (temperature $77 \mathrm{~K}$ ) and subsequent annealing at a temperature of 150-200 K. Metal nanoparticles in film samples can be partially and directly formed during the low-temperature co-condensation and can be additionally formed as a result of the thermal decomposition of the unstable biligand $\pi$-complexes of metal atoms $\mathrm{M}(5 \mathrm{CB})_{2}$ [22]. The metal atoms that are liberated in this process can be localized in the regions of a micro-heterogeneous, disordered glassy liquid crystal matrix that is formed by the alkyl chains of the terminal substituent in $5 \mathrm{CB}$ dimer structures. High molecular 
mobility is maintained in these local micro-regions, even at low temperatures. As a result, the released metal atoms can relatively easily diffuse in the solid-phase matrix and interact with each other, forming nanoparticles that lost the mobility.

The study of selected areas of film samples by electron diffraction has indicated that the resulting metal nanoparticles have a crystal structure with an fcc-cubic lattice that is typical for of silver and copper crystals. The size of silver and copper nanocrystals that are formed in mesogenic CB micro heterogeneous matrices at low temperatures is close to the critical size of metal nanoparticles, at which there is a transition from a crystal-like fcc-cubic structure to quasi-crystalline icosahedral-like structures.

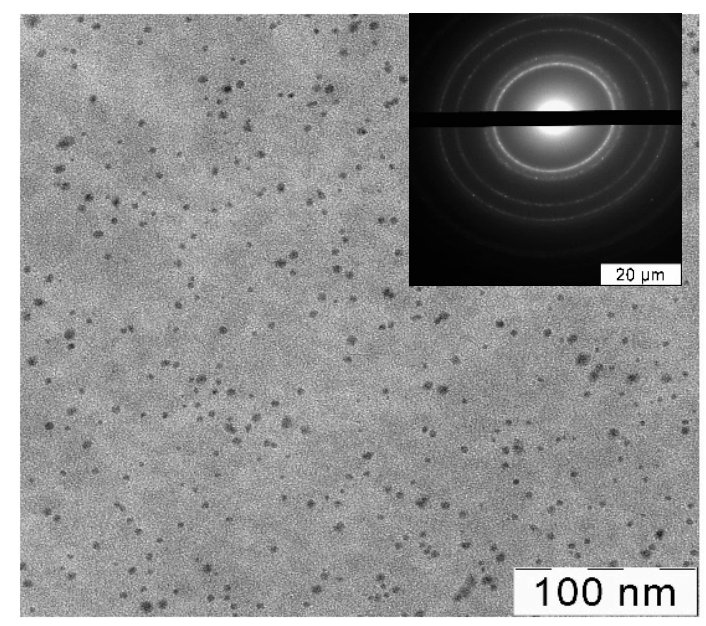

(a)

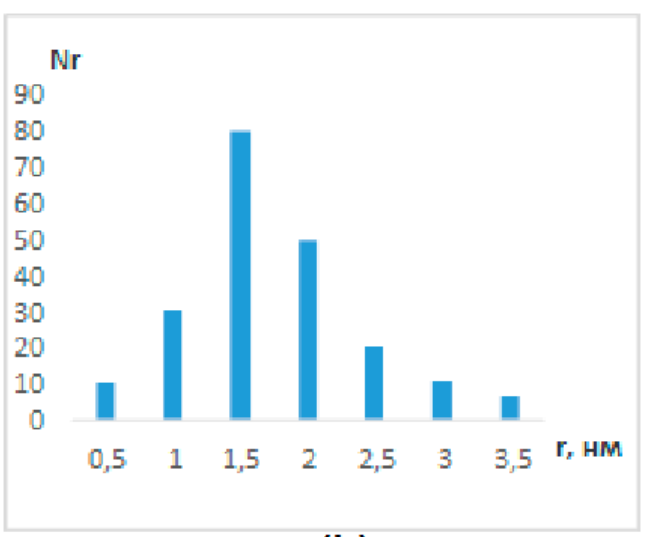

(b)

Figure 6. TEM pictures and electronic diffraction of the samples, including silver nanoparticles that are stabilized in a mesogenic 4-cyano-4'-pentylbiphenyl matrix at $200 \mathrm{~K}$ (a) and a histogram of their size distribution (b).

The heating of the samples of the hybrid metal-cyanobiphenyl nanosystems to temperatures above $200 \mathrm{~K}$ led to the crystallization of a glassy liquid crystal matrix and was accompanied by a sharp increase in molecular mobility. In this case, the fast coalescence of nanoclusters occurred along with the formation of larger metal aggregates that were not registered by the ESR method. Such behavior of the hybrid metal-cyanobiphenyl system was the result of specificity of intermolecular interactions and, as a consequence, the supramolecular organization of the mesogenic cyanobiphenyls matrix and the alteration of its character with temperature.

In cyanobiphenyl molecules, there is a rigid biphenyl core, as well as terminal substituents in the 4 and $4^{\prime}$ positions. On the one hand, there are flexible, non-polar long-chain alkyl or alkoxyl groups, and on the other hand, there are high-polar cyanogroups. The presence of cyanogroups leads to the high polarity of the molecules of cyanobiphenyl as a whole. Intensive dipole-dipole interactions of cyanobiphenyl molecules lead to antiferroelectric ordering, with the formation of dimers arranged on the principle of "head-to-tail." In turn, cyanobiphenyl dimers are capable of further self-organization due to staking interactions between biphenyl rings and other types of intermolecular interactions. In this case, the type of self-organization depends on the sample's prehistory and can change when the temperature changes. The introduction of d-metals $(\mathrm{Ag}$ or $\mathrm{Cu}$ ) into the system, due to the formation of biligand metastable complexes of metal atoms, partially changes the system of intermolecular interactions, breaking the antiferroelectric molecular ordering at long distances.

The complexes were stabilized in the slightly crystallized solid CB matrices up to $200 \mathrm{~K}$. At temperatures close to this value we observed solid-solid phase transition in the CB's solids and the formation of dimeric crystal structures. At temperatures higher than $200 \mathrm{~K}$, the complexes possessed the effective degradation and aggregation of liberated metal atoms in small (1-2 nm in size) global metal nanoparticles stabilized in the metastable solid state which was formed by the CB 
dimers and localized in the area of the more mobile alkyl chains of CB's matrices. When the molecular flows of the metal and the cyanobiphenyl co-condensed on a cold surface at a temperature of $77 \mathrm{~K}$, the atoms and molecules that fell on the surface maintained a high molecular mobility for some time. During this time, some of the metal atoms formed biligand $\pi$ complexes with the cyanobiphenyl molecules. The other metal atoms could aggregate and form nanoscale clusters. Such a mechanism was evidenced by the data obtained by ESR spectroscopy. Cyanobiphenyl molecules that were not included of the biligand $\pi$ complexes formed molecular dimers. However, the time at which the atoms and molecules maintained a high molecular mobility was not sufficient for the self-organization of the cyanobiphenyl molecules and their packing into ordered crystalline phases. As a result of this partial self-organization, we obtained a metastable solid-phase matrix that was partially crystallized and formed nematic-ordered (in the case of $5 \mathrm{CB}$ and $5 \mathrm{OCB}$ ) or smectic-ordered (in the case of $8 \mathrm{CB}$ ) glassy-like phases.

Increasing the temperature led to a partial reorganization of the CB matrix's molecular packing, leading to a rapid increase in molecular mobility. As a result, part of the biligand $\pi$-complexes of the metals with cyanobiphenyl molecules was irreversibly decomposed. Finally, they disappeared at a temperature of about $150 \mathrm{~K}$. The released metal atoms combined with their aggregation and formed spherical metal nanoparticles with a size of 1-2 nm. The aggregation of metal atoms was possible due to their relatively high diffusion over local microblasts of the solid-liquid crystal matrix that was formed by a system of mobile alkyl or alkoxyl substituents of cyanobiphenyl molecules. The average diameter of the resulting nanoparticles $(1-2 \mathrm{~nm})$ was probably determined by the size of these local areas with a high molecular mobility. The metal nanoparticles were stabilized in a the solid-phase matrix. As a result, the system remained stable in the temperature range of 150-200 K, which was confirmed by ESR spectroscopy data.

The heating of the samples that were obtained by the joint co-condensation of the molecular streams of the metal and cyanobiphenyl above $200 \mathrm{~K}$ led to the crystallization of a glassy-like solid-liquid crystal matrix. During the phase transformation, there was a sharp increase in translational molecular mobility. This resulted in the coalescence of small metal nanoparticles into larger aggregates that did not produce signals in the ESR spectra.

The rapid heating of co-condensates to room temperature with a phase transition from crystalline to nematic (in the case of $5 \mathrm{CB}$ and $5 \mathrm{OCB}$ ) or smectic (in the case of $8 \mathrm{CB}$ ) led to the formation of anisometric nanoparticles. For example, in the Ag-5CB system, the growth of highly anisotropic metal nanorods occurred in the crystal matrix in the temperature range of 273-293 K, which was close to the temperature of the crystal-nematic phase transition. The length of these particles $(\mathrm{l}, \mathrm{nm})$ was more than $200 \mathrm{~nm}$, and the width $(\mathrm{d}, \mathrm{nm})$ was about $15 \mathrm{~nm}$. Thus, the anisometric ratio of particles $(\mathrm{l} / \mathrm{d})$ exceeded 10 (Figure 7). The mechanism of such a quasi-one-dimensional growth of metal nanorods is associated with maintaining a high degree of ordering and the simultaneously increasing molecular mobility in the pre-transition region.

The study of the samples containing nanorods by X-ray diffraction demonstrated the crystalline nature of these formations. The texture of the resulting nanorods occurred due to directed growth that was parallel to the crystallographic plane (100) and perpendicular to the plane (111). The reason for this phenomenon may be the fact that the plane (111) of the silver crystal structure was characterized by a hexagonal dense packing, while the plane (100) was characterized by a less dense square packing. In this regard, the intensity of the interactions of the terminal cyano $(\mathrm{CN})$ ligand groups with metal atoms located on the plane (111) was higher than those of the metal atoms located on the plane (100). Due to this circumstance, the fusion of smaller nanocrystals with the formation of larger ones occurred mainly along the plane (100). This resulted in the formation of highly anisometric metal nanorods. After heating the samples to temperatures (or close to these temperatures) of the phase transitions to the liquid crystal states of CB-nematic or smectic types, the rapid aggregation of small nanoparticles was observed with the formation of anisometric nanoparticles and their highly-ordered assemblies 
and nanostructures, with the morphology controlled by the dynamic and structural properties of soft matrix templates of $\mathrm{CB}$.

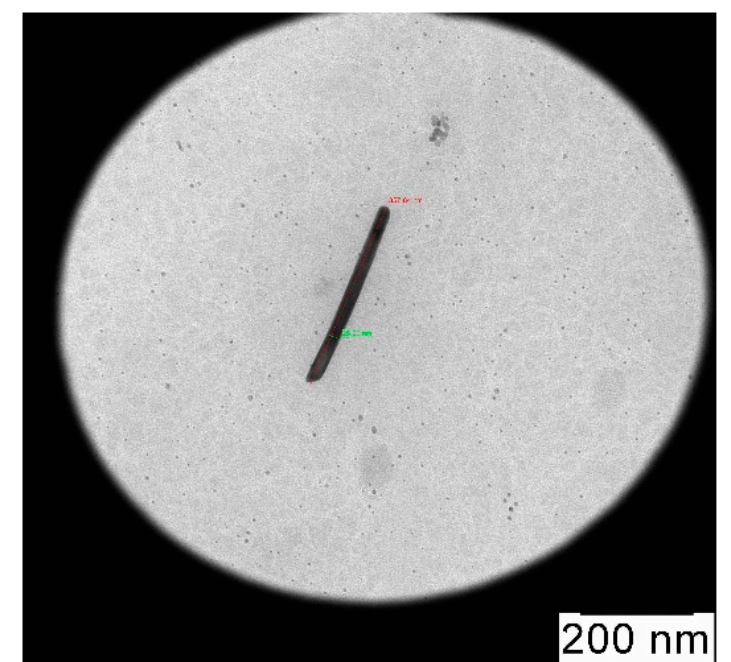

(a)

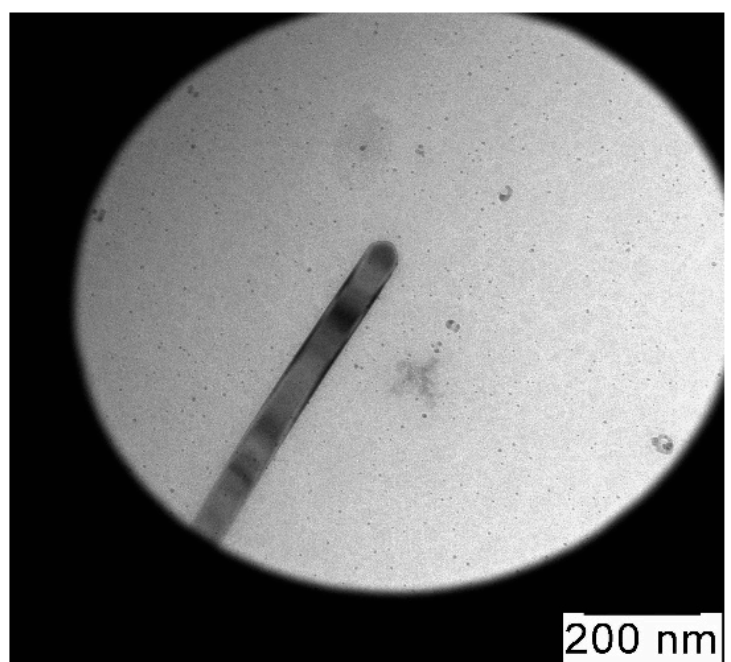

(b)

Figure 7. TEM pictures of silver nanorods growing in a 4-cyano-4'-pentylbiphenyl matrix at 273-290 K (a), at $293 \mathrm{~K}(\mathbf{b})$.

\subsection{Self-Assembling of Metal Nanoparticles in Nematic and Smectic Phases of Mesogenic Cyanoalkylcbiphenyls}

Samples that were obtained by the co-condensation of $\mathrm{Ag}$ or $\mathrm{Cu}$ molecular beams with mesogenic cyanobiphenyls and subsequently controlled annealing were nanohybrid materials that included metal nanoparticles that were stabilized in a matrix of mesogenic ligands, and the morphology of the nanoparticles was largely determined by the processes of the self-ordering of the liquid crystal matrix. This was confirmed by the analysis of the set of data that was obtained by transmission electron microscopy (TEM), the electron diffraction of the selected area (SED), and X-ray diffraction. It was shown that depending on the phase state of the $\mathrm{Ag} / 5 \mathrm{CB}$ and $\mathrm{Ag} / 8 \mathrm{CB}$ hybrid nanosystems, it was possible to form metal nanoparticles of different sizes and morphologies. For example, the rapid heating of samples of the metal-mesogenic co-condensate with a low metal content to a temperature of $313 \mathrm{~K}$, followed by a phase transition to the isotropic phase, led to the formation of spherical metal particles with a characteristic average diameter of $15 \mathrm{~nm}$. The data that were obtained by the method of IR-Fourier spectroscopy indicated the formation of a stabilizing monolayer of alkyl cyanobiphenyl molecules on the surface of the metal nanoparticles due to donor-acceptor interactions of the terminal $\mathrm{CN}$ groups of the ligand with surface metal atoms. Increasing the content of the metal component and conducting the self-assembly processes of nanoparticles in the orientationally-ordered nematic phase led to the formation of anisometric metal nanoparticles with an anisometric factor of $1 / \mathrm{d}$ equal to 3,5 and their highly-ordered ensembles. Atomic force microscopy data, showing the formation of linear aggregates and regularly-ordered ensembles of anisometric nanoparticles in the nematic phase of $5 \mathrm{CB}$ at $300 \mathrm{~K}$, are presented in Figure 8 . A bimodal histogram of the size distribution of the metal nanoparticles for this case is also shown in Figure 8. 


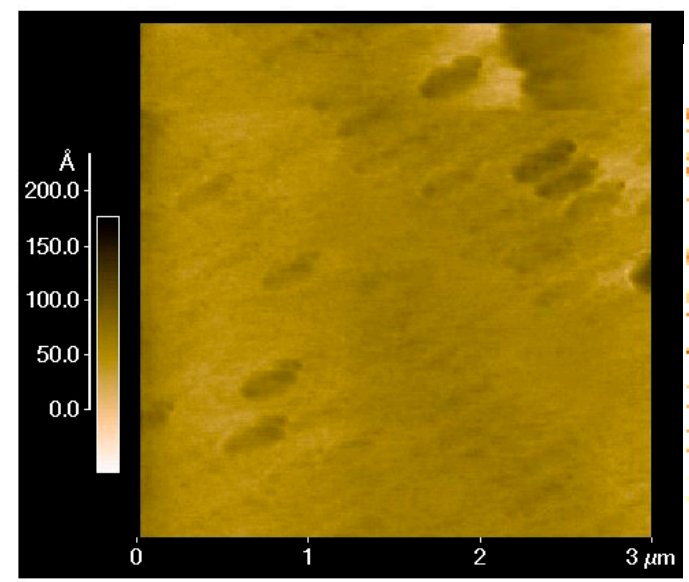

(a)

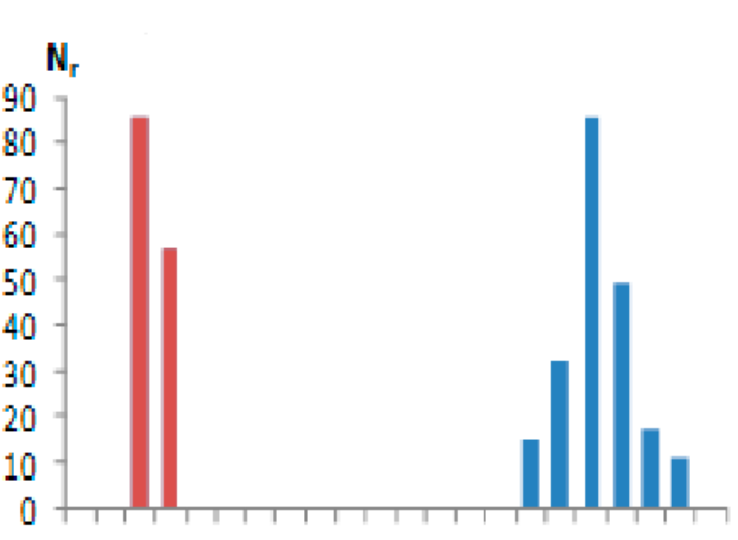

$\begin{array}{lllllllllll}14 & 15 & 16 & 17 & 23 & 29 & 35 & 41 & 44 & 46 & 47,5\end{array}$

Figure 8. Atomic force microscopy (AFM) image (tapping mode) of the sample of the hybrid metal-mesogenic nanosystem "Ag-4-cyano-4'-pentylbiphenyl" (1/10 w/w\%) in nematic phase 4-cyano-4'-pentylbiphenyl (left) (a) and a histogram of the size distribution (right) for the silver nanoparticles (b). Red—cross size of nanoparticles, blue-linear length of nanoparticles.

The use of higher homology 4-cyano-4'-octylbiphenyl (8CB) smectic liquid crystal 8CB as a soft template matrix allowed for the formation of metal nanoparticles in a layered, molecularly-organized system. In this case, the formation of quasi-fractal flat metal aggregates intercalated between smectic layers of $8 \mathrm{CB}$ (Figure 9) was revealed. This was due to the higher interaction energy between the cyanobiphenyl molecules compared to the interaction energy between the cyanobiphenyl molecules and the metal atoms. As a result, the microphase separation of metal atoms and smectic liquid crystal molecules occurred. Silver atoms displaced into the interlayer space that were formed by a system of mobile alkyl substituents could relatively easily diffuse within this space and interact with each other. In this case, the nature of the diffusion processes with the participation of metal atoms was quasi-two-dimensional, which, in the final case, led to the formation of quasi-two-dimensional metal aggregates.

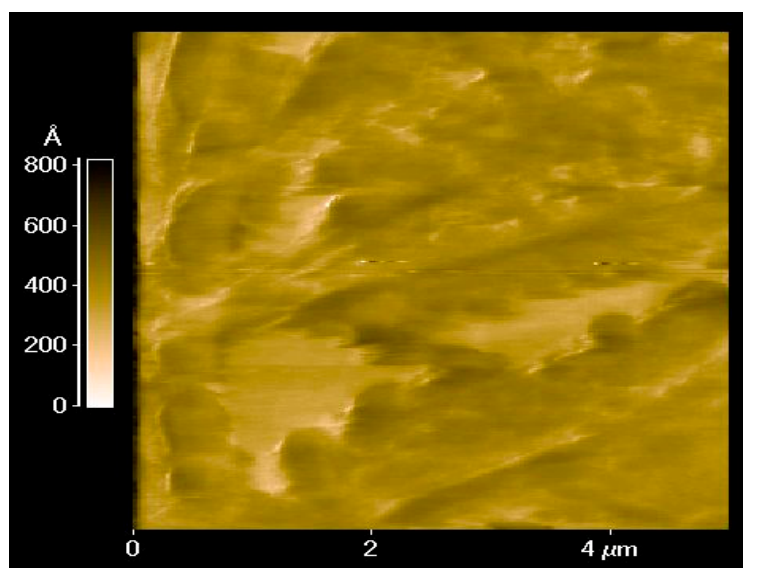

Figure 9. AFM image (tapping mode) of the sample of the hybrid metal-mesogenic nanosystem "Ag-4-cyano-4'-octylbiphenyl" (1/10) in the smectic phase. (The topology of the sample surface (the height) is presented by the color -the color scale is presented at the left part of the picture).

Thus, the processes of the self-organization of the liquid crystal matrix and the associated processes of the aggregation of metal atoms led to the formation of hybrid nanosystems, including metal nanoparticles of different sizes and morphologies that were supramolecularly organized into highly-ordered nanostructures. In particular, these could be anisometric nanoparticles and rod-shaped 
aggregates that stabilized in nematic mesophases, as well as flat two-dimensional metal aggregates that formed in the layered structured smectic phases of long-chain cyanoalkylbiphenyls. At low metal content, in the orientationally-ordered nematic phase, globular metal nanoparticles of smaller size were formed and stabilized. Increasing the concentration of metals contributed to the formation of anisometric rod-shaped metal nanoparticles and their aggregates. The diagram of the structural-phase states of the Ag-5CB hybrid nanosystem, indicating the conditions for the formation of metal nanoparticles with a certain morphology, is shown in Figure 10. This diagram is a summary of the results that were obtained by thermal analysis, poly-thermal polarization spectroscopy, electron diffraction of the sample selected areas (SAED) and atomic force microscopy.

An analysis of the data shown in Figure 10 allowed us to explain the dependence of the size and morphology of metal nanoparticles that were stabilized by the mesogenic CB ligands of the phase state of the mesogenic matrix and the metal concentration. The shape of the metal nanoparticles was the result of competition between three factors - the general tendency to minimize the surface energy of the particle, the energy of intermolecular interactions of liquid crystal molecules with surface metal atoms, and the elastic energy of the liquid crystal. The rapid heating of $\mathrm{Ag} / 5 \mathrm{CB}$ co-condensate samples at speeds exceeding $100 \mathrm{~K} / \mathrm{min}$ to the isotropic phase existence temperatures $(\mathrm{T}>318 \mathrm{~K}$ ) (region I in Figure 10) led to the formation and stabilization of globular nanoparticles with $d=(15 \pm 10) \mathrm{nm}$ in size. The formation of anisometric metal nanoparticles and their orientationally-ordered assemblies occurred in the nematic phase, as seen in region III in Figure 10. In the two-phase region of the coexistence of the isotropic and nematic phases (region II in Figure 10) it was possible to form both isotropic globular particles and anisometric rods.

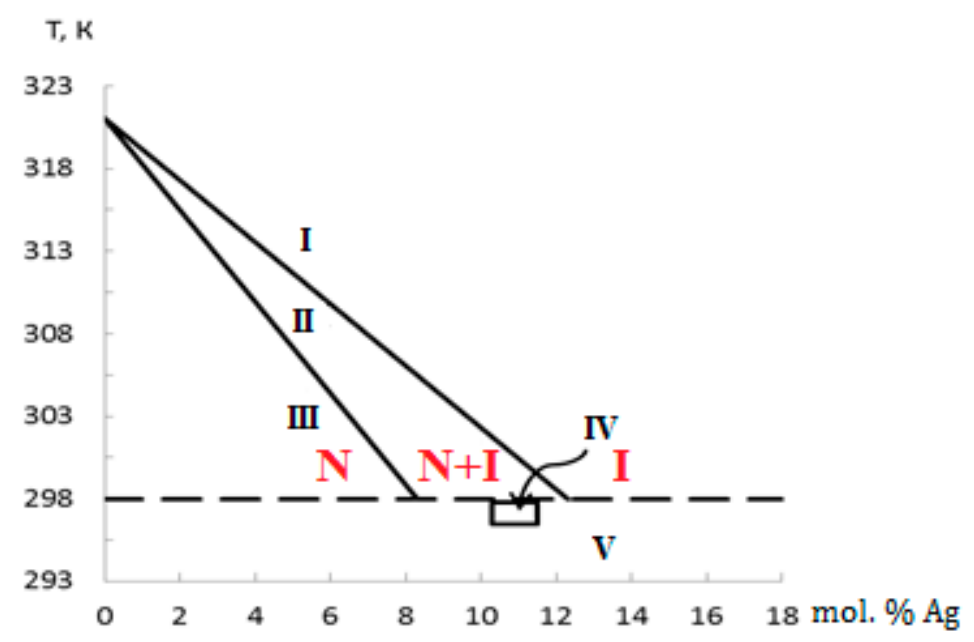

Figure 10. The scheme of phase-structural states for "Ag-4-cyano-4'-pentylbiphenyl" hybrid nanosystem: I-isotropic phase; II—two phase region: isotropic phase including the fluctuations of nematic ordering; III—nematic phase; IV—-the temperature region in nematic phase, which was close to phase transition from crystal to nematic; and V—solid phase at 150-200 K.

The maintaining of the samples at $273-283 \mathrm{~K}$ and the increasing of the metal content in the sample from 1 up to $10 \mathrm{w} / \mathrm{w} \%$ (region IV in Figure 10) led to preferential growth of rod-like metal nanoparticles with $1 /$ d ratios higher than 10 . Small metal nanoparticles of 1-2 $\mathrm{nm}$ in size were formed in the solid phase (region V in Figure 10) at 150-200 K.

\subsection{The Encapsulation of Hybrid Metal-Containing Nanosystems in Polymer Films and Their Optical Properties}

The layer-by-layer co-condensation of the vapors of xylylene monomer, silver atoms and cyanobiphenyl ligand was performed. The poly-para-xylylene monomer was obtained by the pyrolysis of vapors of para-cyclophane (para-di-xylylene) at $923 \mathrm{~K}$. The scheme of the polymerization process is shown in Figure 11. Controlled heating to a temperature of about 150-155 K or the UV irradiation of 
the co-condensates at lower temperatures led to rapid polymerization of the monomer. This resulted in the formation of sandwich-like structures that contained a thin layer of a hybrid nanosystem that was encapsulated in polymer poly-para-xylylene films. The metal content varied from 1 to $10 \mathrm{w} / \mathrm{w} \%$.

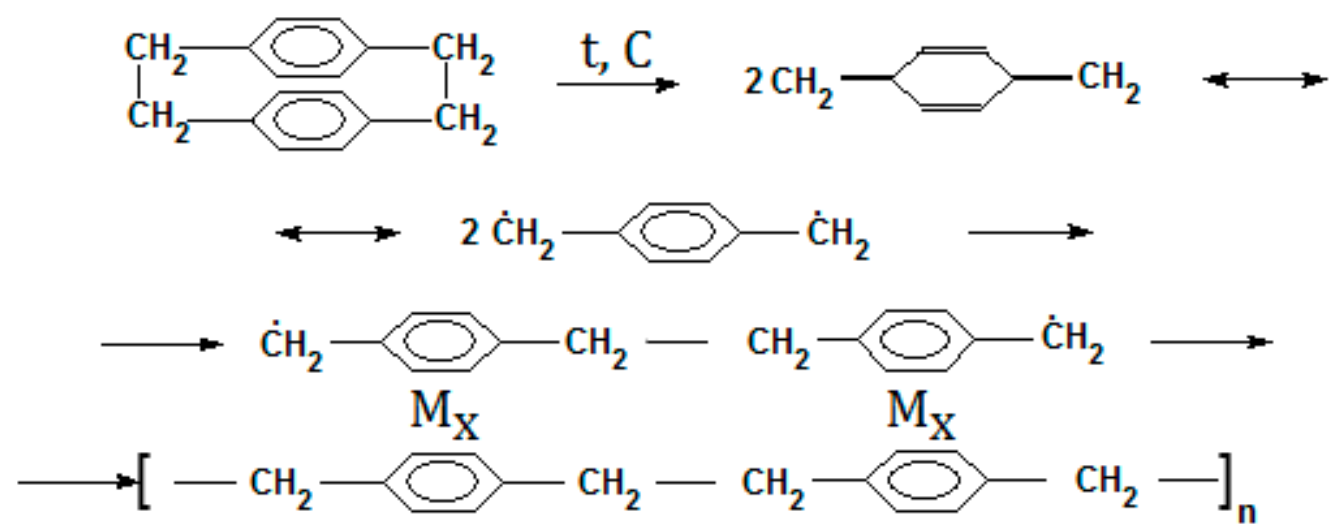

Figure 11. The scheme of the para-xylylene polymerization in triple metal-containing co-condensates.

The results of the study of the samples of the Ag-5CB system encapsulated in poly-para-xylylene films (PPX) by transmission electron microscopy (TEM) showed that the increase in the metal content in the sample up to $10 \mathrm{w} / \mathrm{w} \%$ led to the formation of rod-like metal particles with an anisometric ratio of $1 / \mathrm{d}$ greater than 10 (where $l$ is the length and $d$ is the transverse diameter of the particles). The diffractograms of the Ag-5CB sample (w/w 1:10) that were formed at $200 \mathrm{~K}$ and then annealed to $450 \mathrm{~K}$ are shown in Figure 12. An analysis of the diffractograms allowed us to report on the formation of metal nanoparticles with a cubic fcc structure with a lattice constant $\mathrm{a}=0.408 \mathrm{~nm}$, which is characteristic of crystalline silver. The reflexes that are expressed on the diffractogram were assigned to crystallographic planes (111), (200), (220) and (311). Atomic force microscopy data for an Ag-5CB sample (10 w/w \%) encapsulated in a polymer film indicated the formation of anisometric metal nanoparticles that were stabilized by a liquid crystal matrix and were combined into regular nanostructures (Figure 13). The UV-Vis spectra of film samples are shown in Figure 14. These spectra contain intense bimodal absorption bands that are characteristic of the plasmonic resonance of anisometric silver nanoparticles (Figure 14).

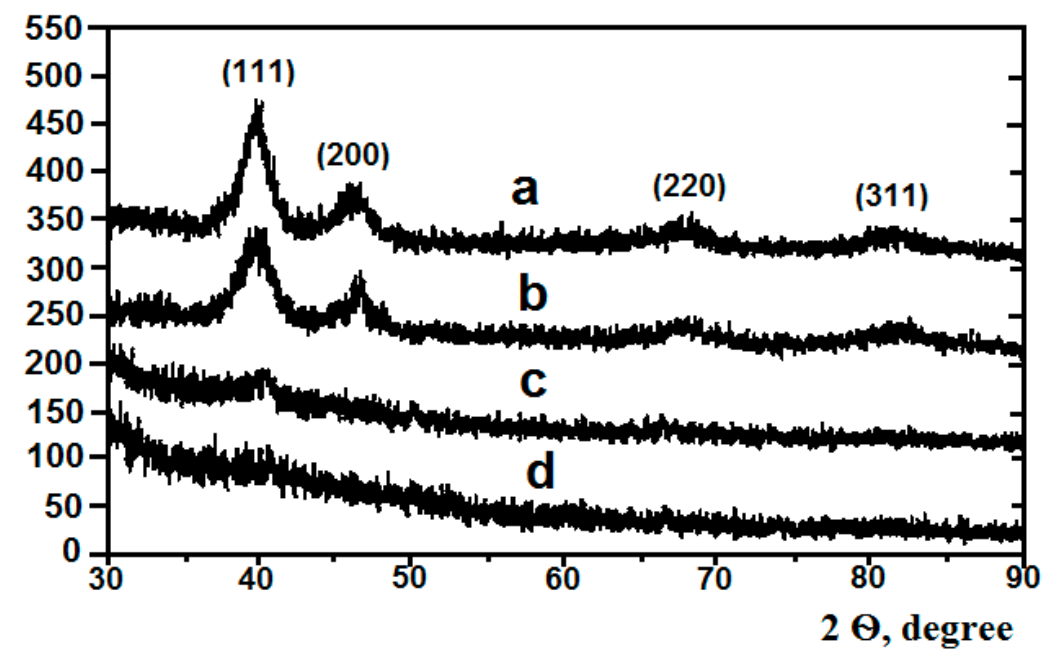

Figure 12. X-ray data for sample of hybrid metal-mesogenic nanosystem Ag/4-cyano-4'-pentylbiphenyl $(1: 10 \mathrm{w} / \mathrm{w} \%)$, encapsulated in poly-para-xylylene (PPX) film at $450 \mathrm{~K}(\mathbf{a}), 300 \mathrm{~K}(\mathbf{b}), 250 \mathrm{~K}$ (c), and $200 \mathrm{~K}$ (d) (Y-axis is the intensity of the signal in related units). 


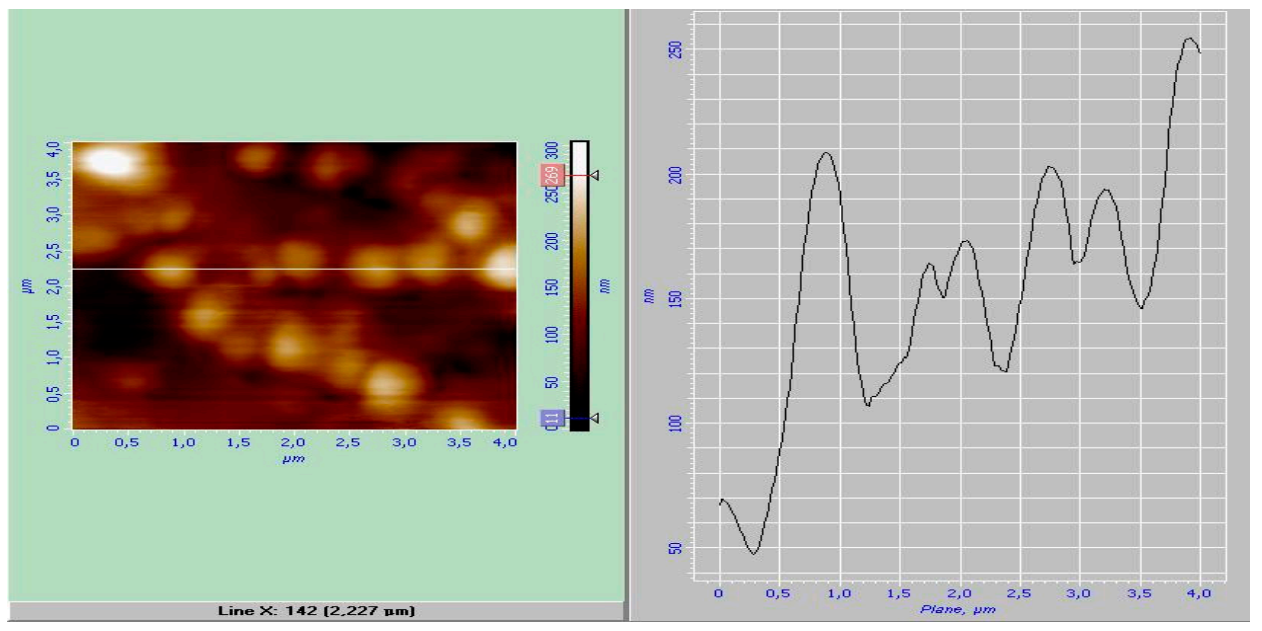

Figure 13. AFM data for the sample of the hybrid metal-mesogenic nanosystem Ag/4-cyano-4'-pentylbiphenyl (1:10 w/w\%) encapsulated in poly-para-xylylene film.

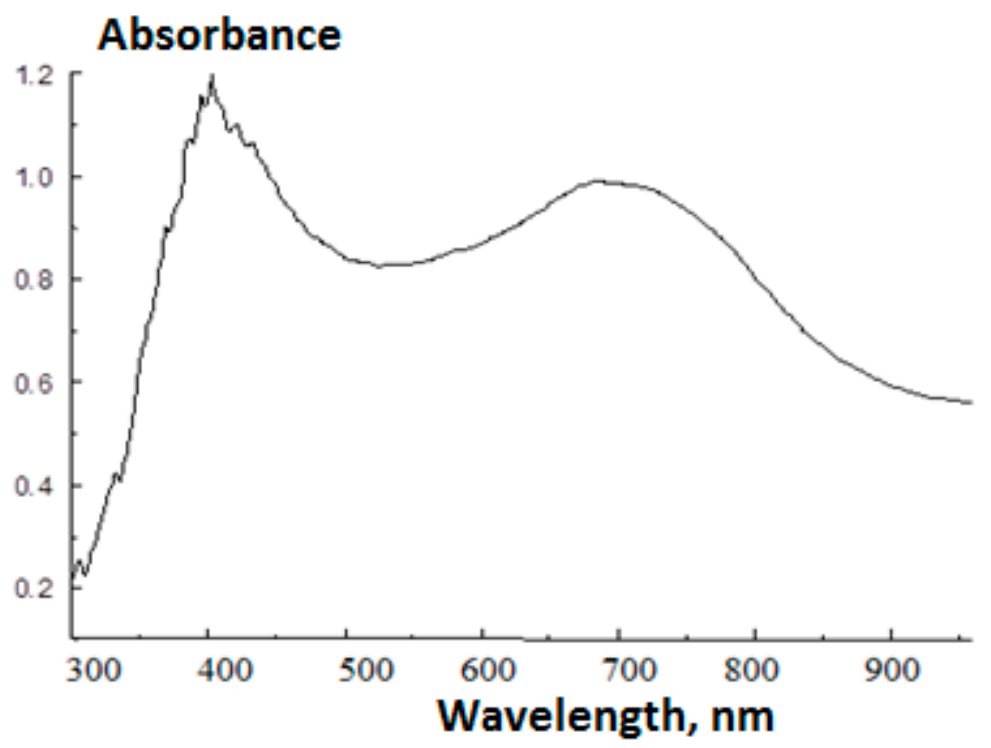

Figure 14. Optical spectra for the sample of the hybrid metal-mesogenic nanosystem Ag/4-cyano-4'-pentylbiphenyl (1:10 w/w\%) encapsulated in poly-para-xylylene film.

The proposed method made it possible to obtain films with a controlled thickness, starting from several molecular layers up to tenths of micrometers. The UV-Vis spectra of the hybrid metal-mesogenic systems encapsulated in PPX films, registered at room temperature $(300 \mathrm{~K})$, contained two plasmonic bands in the range of 420-440 and 650-750 $\mathrm{nm}$ that can be attributed to the transverse and longitudinal modes of the surface plasmonic resonance, respectively. This indicates the formation of anisometric metal nanoparticles and their ordered aggregates. An increase in the metal concentration led to an increase in the anisometric $1 / \mathrm{d}$ ratio. The formation of rod-like metal nanoparticles with an $1 / \mathrm{d}$ ratio of $\approx 10$ led to an increase of plasmonic absorption in the long-wave spectral region $(\lambda \sim 650 \mathrm{~nm})$ [24]. Compared with randomly oriented samples, the imposition of an orienting uniform magnetic field (with an intensity of $\sim 3000 \mathrm{Gs}$ ) led to a five-fold increase in the dichroic ratio of plasmon bands $\mathrm{D}_{\|} / \mathrm{D}_{\perp}$ for the Ag-5CB samples $(1: 10 \mathrm{w} / \mathrm{w} \%)$. The optical properties of the film samples remained stable for several months. 


\section{Conclusions and Outlook}

The proposed work implemented a new approach to the formation of highly-ordered hybrid metal-mesogenic systems. The approach was based on the mesogenic matrix-controlled self-organization of hybrid atomic-molecular systems that are obtained by the low-temperature co-condensation of molecular (atomic) beams of mesogenic components and metals, followed by controlled heat treatment. As a result, hybrid metal-mesogenic nanosystems can be obtained, including metal (silver or copper) nanoparticles that are supramolecularly organized and stabilized by a liquid crystal matrix. Their properties were studied in the temperature range of $77-350 \mathrm{~K}$. The conditions allowed us to obtain metal nanoparticles of different sizes and morphologies have been determined, and methods for creating their ordered nanostructures by the use of the self-organization of liquid crystal matrices have been developed.

The high polarity of cyanobiphenyl molecules that are associated with the presence of a cyanogroup leads to antiferroelectric ordering, which is why the main structural unit of cyanobiphenyl matrices is a dimer that is arranged on the principle of "head-tail." The co-condensation of molecular (atomic) metal streams ( $\mathrm{Ag}$ and $\mathrm{Cu}$ ) and mesogenic component $(5 \mathrm{CB}, 5 \mathrm{OCB}$, and $8 \mathrm{CB}$ ) on a surface that is cooled with liquid nitrogen leads to the formation, at the first stage, of the metastable biligand complexes of metal atoms with cyanobiphenyls stabilized in ligand matrices, representing nematic or smectic glass. The complexes that are formed as a result of low-temperature condensation are metastable and, with each step, increase in temperature within 77-150 K; a certain part of the complexes decomposes, and, at temperatures above $150 \mathrm{~K}$, their concentration becomes lower than the sensitivity limit of an ESR device. The released metal atoms are initially combined to form small metal clusters from which nanoparticles are then formed. The growth of nanoparticles is accompanied by their additional structuring under the action of an anisotropic microheterogenic liquid crystalline matrix. The processes of the self-assembly and aggregation of metal atoms and small metal clusters lead to the formation of metal nanoparticles and their ordered aggregates. These processes are controlled by the structural and dynamic properties of the mesogenic matrix. The nature of these properties changes smoothly with temperature change, but it undergoes radical changes during phase transitions. The structure and physical properties of hybrid nanosystems are determined by the competition of matrix self-organization processes, metal aggregation processes, and the system of intermolecular interactions between metal particles of different sizes with ligand molecules and their molecular associates.

In the UV-Vis spectra of hybrid nanosystems, both bands corresponding to the surface plasmon resonance of individual silver nanoparticles and the bands with complex shapes that are caused the surface plasmon resonance of hybrid nanostructures and highly-ordered super-lattices of metal nanoparticles appear. The intensity ratio of these bands is determined by the metal content and temperature prehistory of the samples. Such hybrid nanostructures are promising for creating new optical and electro-optical devices with controlled light absorption and reflection properties. Such systems can be very promising for various applications in nanoelectronics and electro-optics, and they have unusual optical and sensory properties. The integration of silver nanoparticles of exact sizes and shapes with biological molecules and their aggregates opens new perspectives for obtaining of new bio-compactable instruments for the imaging and sensing of biological objects in living organisms and targeted medical therapy.

Supplementary Materials: The following are available online at http://www.mdpi.com/2073-4352/10/2/77/s1, Figure S1: The scheme of cryostat for ESR-spectroscopy, Figure S2: The electron density distribution in the ground state of biligand $\operatorname{Ag}(5 \mathrm{CB})_{2}$ complex for two electronic configurations: charge transfer configuration(A) and neutral biligand $\pi$-complex(B) of silver atom.

Author Contributions: Conceptualization, methodology, writing of original draft and editing-T.I.S., Spectral Investigations and Original Figures Preparation-Y.N.M. All authors have read and agreed to the published version of the manuscript.

Funding: T.I.S. and Y.N.M. thanks Russian Foundation of Basic Research (grant No 18-03-00730-a) for financial support. 
Acknowledgments: T.I.S. and Y.N.M. thank A.V. Bochenkova for quantum chemistry modelling of bi-ligand complex $\mathrm{Ag}(5 \mathrm{CB})_{2}$ structure, S.S. Abramchuk, Centre of Electronic Microscopy of M.V. Lomonosov Moscow State University for TEM and SAED measurements and A.S. Tikhomirov, NT MDT for AFM measurements.

Conflicts of Interest: The authors declare no conflicts of interest.

\section{References}

1. Blinov, L.M. Structure and Properties of Liquid Crystals; Springer: Dordrecht, The Netherlands, 2011; p. 439.

2. Lagerwall, J.P.F.; Scalia, G. A new era for liquid crystal research: Applications of liquid crystals in soft matter nano-, bio- and microtechnology. Curr. Appl. Phys. 2012, 12, 1387-1412. [CrossRef]

3. Schmidt, G. Nanoparticles: From Theory to Applications, 2nd ed.; Wiley-VCH: Weinheim, Germany, $2010 ;$ p. 533.

4. Klabunde, K.J.; Richards, R.M. Nanoscale Materials in Chemistry, 2nd ed.; John Wiley \& Sons: New York, NY, USA, 2009; p. 792.

5. Green, A. Theory and Application of Nanoparticles; NY Research Press: New York, NY, USA, 2015; p. 218.

6. Sergeev, G.B.; Klabunde, K.J. Nanochemistry, 2nd ed.; Elsevier: Amsterdam, The Netherland, 2013; p. 249.

7. Talapin, D.V.; Lee, J.; Kovalenko, M.V.; Shevchenko, E.V. Prospects of Colloid Nanocrystals for Electronic and Optoelectronic Applications. Chem. Rev. 2010, 110, 389-458. [CrossRef] [PubMed]

8. Boles, M.A.; Engel, M.; Talapin, D.V. Self-Assembly of Colloidal Nanocrystals: From Intricate Structures to Functional Materials. Chem. Rev. 2016, 116, 11220-11289. [CrossRef] [PubMed]

9. Sugimoto, T. Monodispersed Particles, 2nd ed.; Elsevier: Amsterdam, The Netherlands, 2019; p. 850.

10. Jane, P.K.; Huang, X.; El-Saed, I.H.; El-Saed, M.A. Noble metals on the nanoscale: Optical and photothermal properties and some applications in imaging, sensing, biology and medicine. Acc. Chem. Res. 2008, 41, 1578-1586. [CrossRef] [PubMed]

11. Millstone, J.E.; Hurst, S.J.; Metraux, G.S.; Cutler, J.I.; Mirkin, C.A. Colloidal gold and silver triangular nanoprisms. Small. 2009, 5, 646-664. [CrossRef] [PubMed]

12. Wang, L.; Xu, L.; Kuang, H.; Xu, C.; Kotov, N.A. Dynamic nanoparticle assemblies. Acc. Chem. Res. 2012, 45, 1916-1926. [CrossRef] [PubMed]

13. Grzekzak, M.; Vermant, J.; Furst, E.M.; Liz-Marzan, L.M. Directed Self-Assembly of Nanoparticles. ACS Nano 2010, 4, 3591-3605. [CrossRef] [PubMed]

14. Nie, Z.; Petukhova, A.; Kumacheva, E. Properties and emerging applications of Self-assembled Structures made from inorganic nanoparticles. Nat. Nanotechnol. 2010, 5, 15-25. [CrossRef] [PubMed]

15. Macfarlane, R.J.; Lee, B.; Matthew, R.J.; Harris, N.; Schatz, G.C.; Mirkin, C.A. Nanoparticle superlattice engineering with DNA. Science. 2011, 334, 204-208. [CrossRef] [PubMed]

16. Dong, A.; Chen, J.; Vora, P.M.; Kikkawa, J.M.; Murray, C.B. Binary nanocrystal superlattice membranes self-assembled at the liquid-air interface. Nature 2010, 446, 474-478. [CrossRef] [PubMed]

17. Okuda, M. $\mathrm{Fe}_{3} \mathrm{O}_{4}$ nanoparticles: Protein mediated crystalline superstructures. Nanotechnology 2012, $23,415601$. [CrossRef] [PubMed]

18. Grill, L.; Dver, M.; Lafferenz, L.; Persson, M.; Peters, M.V.; Hecht, S. Nano-architectures by covalent assembly of molecular building blocks. Nature Nanotechnol. 2007, 2, 687-691. [CrossRef] [PubMed]

19. Stoeva, S.; Klabunde, K.J.; Sorensen, C.M.; Dragieva, I. Gram-scale synthesis of monodisperse gold colloids by the solvated metal atom dispersion method and digestive ripening and their organization into two- and three dimensional structures. J. Am. Chem. Soc. 2002, 124, 2305-2308. [CrossRef] [PubMed]

20. Pileni, M.-P. Self-assembly of inorganic nanocrystals: Fabrication and collective intrinsic properties. Acc. Chem. Res. 2007, 40, 685-693. [CrossRef] [PubMed]

21. Shabatina, T.I.; Sergeev, G.B. Cryochemistry of Nanometals, Chapt.11. In Polymer Thin Films; Hasmin, A.A., Ed.; InTechOpen Ltd.: London, UK, 2010; pp. 185-196.

22. Shabatina, T.I. Cryoformation of hybrid metal-mesogenic nanosystems. Mol. Cryst. Liq. Cryst. 2011, 545, 1268-1275. [CrossRef]

23. Shabatina, T.I.; Belyaev, A.A.; Morosov, Y.N. Self-Organisation Processes and Phase-Structural States in Hybrid Metal-Mesogenic Nanosystems. Mol. Cryst. Liq. Cryst. 2016, 632, 70-78.

24. Shabatina, T.I.; Belyaev, A.A.; Sergeev, G.B. Self-assembled nanostructures in Silver/Thiocholesterol and Silver/Cholesterol Systems. Bio Nano Science. 2013, 5, 289-294. [CrossRef] 
25. Shabatina, T.I.; Morosov, Y.N.; Timoshenko, V.A. The ESR-Study of Chemical Interaction in Triple Solid "Ag-CCl 4 -5CB" Co-Condensate Mesogenic System. Mol. Cryst. Liq. Cryst. 2005, 440, 325-333. [CrossRef]

26. Wessel, G.; Lew, H. Hyperfine Structures of Silver and Gold by the Atomic Beam Magnetic Resonance Method. Phys. Rev. 1953, 92, 641-646. [CrossRef]

27. Howard, J.A.; Joly, H.A.; Mile, B. Electron paramagnetic resonance spectra and structures of $\mathrm{Cu}_{2}\left[\mathrm{C}_{2} \mathrm{H}_{4}\right]$, $\mathrm{Cu}\left[\mathrm{C}_{2} \mathrm{H}_{4}\right]_{2}$ and $\mathrm{Cu}\left[\mathrm{C}_{2} \mathrm{H}_{4}\right]_{3}$ in hydrocarbon matrices. J. Phys. Chem. 1990, 94, 1275-1279. [CrossRef] 